\title{
PRIMARY OPEN ANGLE GLAUCOMA AND HYPOTHYROIDISM: CHANCE OR TRUE ASSOCIATION?
}

\author{
J. T. GILLOW, P. SHAH and E. C. O'NEILL \\ Birmingham
}

\begin{abstract}
SUMMARY
The prevalence of hypothyroidism in British patients with primary open angle glaucoma (POAG) was examined. A recently reported study from Montreal had shown a significant increase $(p<0.004)$ in biochemical hypothyroidism $(23.4 \%)$ in a population of 64 POAG patients compared with controls $(4.7 \%)$. Mechanisms for a possible causal association between the two diseases are discussed, including mucopolysaccharide deposition in the trabecular meshwork and vasculopathy altering ocular bloodflow. Reports of improved glaucoma control following treatment of hypothyroidism are discussed. This study examined 100 consecutive patients with POAG in a specialist glaucoma clinic. All patients were questioned regarding symptoms of thyroid dysfunction and previous thyroid disease. All patients not already taking thyroxine underwent an assay of thyroid stimulating hormone. The $4 \%(95 \%$ CI $1.1-9.4 \%)$ prevalence of overt hypothyroidism in our study shows no clinically significant increase either over controls in the Montreal study or over our local population. We conclude that in our local population there is no evidence for a clinically important association of hypothyroidism with glaucoma.
\end{abstract}

A recent study ${ }^{1}$ found a statistically significant association between hypothyroidism and primary open angle glaucoma (POAG). Other reports have reached conflicting conclusions. This study was designed to test whether a clinically significant association was present in a UK glaucoma clinic population.

\section{METHODS}

Patients attending a glaucoma special clinic were studied for evidence of hypothyroidism. One hun-

Correspondence to: Timothy Gillow, MRCP, FRCOphth, Birmingham and Midland Eye Centre, Dudley Road, Birmingham B18 7QH, UK. dred consecutive patients were recruited and gave informed consent for the study. Patients were questioned regarding symptoms of thyroid dysfunction and previous thyroid disease and were examined for evidence of clinical hypothyroidism.

All the patients not already taking thyroxine underwent a standardised assay of thyroid stimulating hormone (TSH). The immunoradiometric TSH assay used in this study (Kodak hsTSH Assay) is highly sensitive for hypothyroidism since the level is always markedly raised in primary hypothyroidism. The sensitivity of the test is $0.013 \mu \mathrm{IU} / \mathrm{ml}$ and is defined as the minimum concentration of TSH which can be statistically distinguished from the zero standard in the assay. Patients with a TSH level greater than $5 \mu \mathrm{IU} / \mathrm{l}$ were tested for free $\mathrm{T}_{4}$ and underwent a repeat test of the TSH level.

Patients with evidence of hypothyroidism were divided into two groups: overt hypothyroidism and subclinical hypothyroidism. A diagnosis of 'overt hypothyroidism' was made if the clinical picture was consistent with the biochemical findings of raised TSH with reduced free $\mathrm{T}_{4}$. A raised TSH level without reduced free $T_{4}$ has been labelled 'subclinical hypothyroidism'. Patients with evidence of overt or subclinical hypothyroidism were assessed by an endocrinologist.

\section{RESULTS}

In the study population there were 52 men and 48 women with a mean age of 65 years. There were 85 patients with POAG and 15 glaucoma suspects requiring treatment. Eighty-eight of the subjects were of Caucasian origin, 11 Afro-Caribbean and 1 Asian.

Of the 100 patients interviewed and examined in the glaucoma group, 3 were on thyroxine replacement. A fourth had a history of thyroiditis but was clinically and biochemically euthyroid without 
treatment. Patients taking thyroxine were excluded from the biochemical testing.

Four patients had a raised TSH level ( $>5 \mu \mathrm{IU} / \mathrm{l})$ on one occasion. When a raised TSH value was found the test was repeated together with a thyroid hormone assay. Only 2 of the 100 patients tested had a raised TSH level on two occasions. One of these was clinically and biochemically hypothyroid, had a low free $\mathrm{T}_{4}$ and was started on thyroxine replacement. The other was an 80-year-old woman who was clinically euthyroid, had a mildly elevated TSH level, normal free $T_{3}$ and $T_{4}$, and in whom further intervention was deemed inappropriate. Only 1 patient with previously undiagnosed hypothyroidism requiring treatment was identified by this study. The rate of overt hypothyroidism including those already on thyroxine replacement was $4 \%$. The rate of subclinical hypothyroidism defined by a single raised TSH value and a clinically euthyroid state with normal free $\mathrm{T}_{4}$ was $3 \%$.

\section{DISCUSSION}

An association between hypothyroidism and POAG has been postulated since the 1920 s. Hertel ${ }^{2}$ reported two patients in whom the intraocular pressure fell after treatment of their hypothyroidism. It has been proposed that in the untreated hypothyroid state hyaluronic acid accumulates excessively in the trabecular meshwork and/or aqueous causing an obstruction to outflow. ${ }^{1}$ An increased facility of outflow has been reported in a patient with open angle glaucoma after treatment of hypothyroidism. ${ }^{3}$

This study reports a rate of overt hypothyroidism of $4 \%$ and subclinical hypothyroidism of $3 \%$. The large Birmingham thyrotrophin study provides normative data. This study targeted all patients aged 60 years or over who were registered with a single general practice. Of those invited to take part, $89.8 \%$ (1210 subjects) did so. This large community-based study $^{4}$ differed from our study population in certain respects. The community study was restricted to the over-60 age group whilst the glaucoma study population had 22 subjects under 60 years of age (mean age 65 years; SD 13.7); the glaucoma study population also had an excess of males and nonCaucasians. The Birmingham thyrotrophin study, using the same definitions of overt and subclinical hypothyroidism, found that $2.9 \%$ had overt hypothyroidism and $7.8 \%$ had subclinical hypothyroidism. The small excess of overt hypothyroidism in the glaucoma population (4\% with $95 \%$ CI $1.1-$ $9.4 \%$ ) is not statistically significant (Fisher's exact test, $p=0.53$ ). This clinically insignificant excess of hypothyroidism would require a very large study population to give statistical significance to the difference. To achieve an $80 \%$ power to detect this difference as statistically significant would require in excess of 1700 subjects. Whilst being unable to prove there is no association between hypothyroidism and glaucoma, we have demonstrated that there is no clinically significant association.

Smith et al. ${ }^{1}$ found an association between hypothyroidism and glaucoma. They found a $23.4 \%$ prevalence of hypothyroidism in their clinic POAG population, which was a highly significant increase compared with the $4.7 \%$ in their control population $(p<0.004)$. Their study was the first in the literature to use the sensitive TSH assay to screen a glaucoma clinic population. This important finding, if reproducible, would have major consequences. Our study used similar methodology based on TSH screening and was of similar sensitivity to the Montreal study, but despite this we were unable to demonstrate an increased rate of hypothyroidism in our local glaucoma population.

\section{CONCLUSION}

This study failed to demonstrate a clinically significant increase in hypothyroidism in our clinic primary open angle glaucoma population. Although a causal link between hypothyroidism and glaucoma is an attractive hypothesis, it is not evident in our local glaucoma population. This is an important negative finding to report.

Key words: Glaucoma, Hypothyroidism.

\section{REFERENCES}

1. Smith KD, Arthurs BP, Saheb N. An association between hypothyroidism and primary open angle glaucoma. Ophthalmology 1993;100:1580-4.

2. Hertel G, Eineges über der Augendruck und Glaukom. Klin Monatsbl Augenheilkd 1920;64:390-2.

3. Smith KD, Tevaarwerk GJM, Allen LH. An ocular dynamic study supporting the hypothesis that hypothyroidism is a treatable cause of secondary open angle glaucoma. Can J Ophthalmol 1992;27:341-2.

4. Parie JV, Franklyn JA, Cross KW, Jones SC, Shepherd MC. Prevalence and follow-up of abnormal thyrotrophin $(\mathrm{TSH})$ concentrations in the elderly in the United Kingdom. Clin Endocrinol 1991;34:77-83. 